\title{
Performance Evaluation of QoS Parameters during WiMAX to WiMAX Handoff using NS2 and QualNet
}

\author{
Sahana Bhosale \\ Dept. of Electronics \& Telecommunication Engg, \\ Bharati Vidyapeeth's College of Engg for Women, \\ Pune, India
}

\author{
R.D. Daruwala \\ Department of Electronics Engineering, \\ Veermata Jijabai Technological Institute (VJTI) \\ Mumbai, India
}

\begin{abstract}
Terminal mobility with Quality of Service (QoS) can be provided through efficient and seamless handoffs and handoff management procedures in $4 \mathrm{G}$ wireless access networks. The primary objective of this work is to analyze the handoff management procedures within a WiMAX access networks and to evaluate its impact on different QoS metrics such as Packet Loss, Throughput and End-to-End Delay. The performance analysis is done using two simulators namely Network Simulator and QualNet. Simultaneously the work also evaluates the reliability of ns2 by comparing the results with QualNet so as to ascertain its usability for vertical handoff management procedures. This type of experimentation is very useful to help students understand the handoff concept in a very simple and lucid manner
\end{abstract}

\section{Keywords}

WiMAX, handoff, throughput, delay, packet loss, ns2, QualNet

\section{INTRODUCTION}

Handoff or handover is a process of changing Mobile Node's (MN) Point of Attachment (PoA) when the Received Signal Strength (RSS) of the MN falls below a pre-defined threshold value. Traditional or conventional handoffs also known as horizontal handoffs (HHOs) occur between the same type of access networks (WiFi-WiFi or GSM-GSM) and are fundamentally based on RSS [1].

HHO takes place if the MN senses a RSS $_{\text {new }}$ which is higher than the current RSS ( $\mathrm{RSS}_{\text {curr }}$ ) and $\mathrm{RSS}_{\text {curr }}$ is less than the predefined threshold value [2]. In addition to threshold value, Hysteresis margin can also be set so as to take handoff based on both predefined values. Sometimes, a dwell timer is also considered alongwith Hysteresis and threshold. Timer starts when the condition to take a handoff is met and MN performs a handoff if the condition holds true for the entire duration of dwell timer.

Thus, RSS is an indicator of the usability of the network and the only criteria of handoff decisions in case of HHOs. Relative RSS is not applicable in case of handoffs between heterogeneous networks, such type of handoffs are also called as vertical handoffs. RSS from heterogeneous networks cannot be compared directly due to the disparity of the technologies involved, in such cases, other network parameters such as bandwidth, handoff signalling delay, initial connection delay, usage cost, network traffic load etc. The primary difference between VHO and $\mathrm{HHO}$ lies in its symmetry. $\mathrm{HHO}$ is considered to be a symmetric process whereas VHOs are asymmetric in nature [3].
This paper presents handoff procedure between WiMAX to WiMAX and performance analysis of QoS metrics during handoff using ns2 and QualNet [4,5].

The paper is organized as follows. Section 2 describes simulators such as ns2 and QulaNet and their implementations. Section 3 discusses WiMAX to WiMAX handoffs which include actual handoff procedure, simulation scenario and graphical performance analysis of handoff metrics using ns2 and QualNet. Finally, section 4 concludes the article.

\section{SIMULATORS}

Most commonly used tools for the implementation and evaluation of horizontal and vertical handoffs are OPNET, OMNET, QualNet and Network Simulators (ns2). MATLAB has also been effectively used by large number of handoff algorithms and is one of the very simple and , effective tool to evaluate the mathematical models of horizontal and vertical handoffs. But ns2 and QualNet are considered to be powerful solutions to model different access technologies. It supports WiMAX (802.16), Wi-Fi (802.11), UMTS, Ethernet (802.3), CDMA 2000 and Bluetooth $[4,5]$.

\subsection{NS2 Implementation}

Network Simulator version 2.34 is used on Linux Ubuntu 10.04 and the networks are designed using the tcl scripting language. This version directly implements $\mathrm{WiFi}$ modules as they are available by default and tcl script is executed without any changes in ns-2.34 version. But WiMAX modules are not available by default and WiMAX extension modules are to be installed through patch files.

According to the IMT2000 QoS Classes and Requirements (3GPP-TS 23.107), Conversational, Streaming, Interactive and Background type of traffic classes are considered for experimentation and simulation purpose. Conversational class which applies to real time speech and also real time video and is highly delay sensitive. Streaming traffic class applies for real time video and audio and is a one way transport. Bandwidth and jitter are important for this class and delay variation of the endto-end real time data flow must be minimal for reasonable QoS resolution. Interactive class applies when the end-user is online requesting data from remote server, e.g Web browsing. Latency and bandwidth are not crucial but it requires low Bit Error Rate (BER). Background class applies when the end-user sends and receives data-files in the background such as delivery of e-mails, file downloads and SMS. This class is not time sensitive but needs low BER [6].

However, the mechanism to ensure QoS for real-time applications has not been established yet for handoff scenarios and hence, the applications must tolerate some degradation of QoS in terms of packet loss, throughput, handoff delays, handoff 
failures and unnecessary handoffs for the traffic transmitted over the networks [7].

Traditionally, UDP has been used as a transport layer protocol for real-time applications instead of TCP. UDP is a much simpler protocol without connection setup delays, flow control, and retransmission, providing applications with a rawer interface to the network. Hence, UDP meets the needs of delay-sensitive real-time applications that can implement their own flow control and retransmission schemes.

UDP AGENTS: UDP agents are implemented in udp. $\{\mathrm{cc}, \mathrm{h}\}$. A UDP agent accepts data in variable size chunks from an application and segments the data if needed. UDP packets also contain a monotonically increasing sequence number and an RTP timestamp. Although real UDP packets do not contain sequence numbers or timestamps, this sequence number does not incur any simulated overhead, and can be useful for trace file analysis or for simulating UDP-based applications [4].

The default maximum segment size (MSS) for UDP agents is 1000 byte:

Agent/UDP set packetSize_1000; \#max segment size

The following commands are used to setup UDP agents in simulation scripts:

set udp0 [new Agent/UDP]

This creates an instance of the UDP agent.

\$ns_attach-agent <node><agent>

This is a common command used to attach any <agent> to a given $\langle$ node $>$.

\$traffic-gen attach-agent <agent>

This is a class Application/Traffic/<traffic type> method which connect the traffic generator to the given <agent>. For example, if we want to setup a CBR traffic flow for the udp agent, udp1, the following commands are given:

set cbr1 [new Application/Traffic/CBR]

\$cbr1 attach-agent \$udp1

\$ns_connect $<$ src-agent $><$ dst-agent $>$

This command sets up an end-to-end connection between two agents (at the transport layer).

TCP AGENTS: TCP is a dynamic reliable congestion control protocol. It uses acknowledgements created by the destination to know whether packets are well received; lost packets are interpreted as congestion signals. TCP thus requires bidirectional links in order for the acknowledgements to return to the source. There are a number of variants of the TCP Protocol. Running an TCP simulation requires creating and configuring the agent, attaching an Application-level data source (a traffic generator), and starting the agent and the traffic Generator [4].

There are two major types of TCP agents: one-way agents and a two-way agent. One way agents are further subdivided into a set of TCP senders (which obey different congestion and error control techniques) and receivers (sinks). The two-way agent is symmetric in the sense that it represents both a sender and receiver. It is still under development. The following commands sets up an end-to-end connection between two TCP agents (at the transport layer) [4].

set src [new Agent/TCP] ; \# create agent

set sink [new Agent/TCP/TCP_SINK]; \# create agent \$ns_attach-agent \$node_(s1) \$src ; \# bind src to node

\$ns_attach-agent \$node_(k1) \$sink; \# bind sink to node

\$src set fid_ 0 ;

\# set flow ID field

$\$$ sink set fid_ 0 ;

\# set flow ID field

\$ns_connect \$src \$sink; \# active connection src to sink

\$ns_at \$start-time "\$ftp start" ; $\quad$ \# start ftp flow

\subsection{QualNet Implementation}

QualNet is a comprehensive tool for modeling large wired and wireless networks. It uses simulation to predict the behaviour and performance of simulated networks so as to improve their design and operations. QualNet is not an open source software as ns2. QualNet is developed by Scalable Network Technologies; Inc. QualNet comes with different libraries such as Wi-Fi, WiMAX, CDMA, GSM etc. This experimentation uses WiMAX libraries. It is very simple to design a scenario in Qualnet as it is a GUI based simulator. Here user can just drag and drop various components from the toolset which are to be connected in the network to be designed. After placing the nodes, the user can connect the different components and links as required. User can define the specifications of the components by defining their properties, specifying the type of network to be used i.e 802.11 or 802.16 , the protocol, mobility of a node, characteristics of the network etc [5].

\section{WiMAX to WiMAX HORIZONTAL HANDOFF}

\subsection{Handoff Procedure}

During normal operation of WiMAX, the MN is always scanning the RSS from the current serving BS. RSS from the neighbouring Base Stations (BSs) can be received through neighbour advertisement messages. This helps in selecting the new target BS in case RSS from the serving BS drops below the predefined threshold value. After gathering information about the neighbouring BSs, MN sends a scanning interval request message to the serving BS if the conditions defined for triggering handoff are satisfied [8].

The HHO process can be network-controlled or mobilecontrolled. The very first step of handoff is the handover preparation stage. It is executed by transmitting a handoff request message; a MN_MNHO-REQ. $M N$ will send this message if the handover is mobile-controlled and BS would send MN_BSHO-REQ in case of network-controlled handoff. MN_MNHO-REQ is transmitted to all neighbouring target BSs by service access network of serving BS. This message includes flow management information required for re-establishment of the connections after the execution of handoff. At this stage, connectivity may get established between the target service network and the source service network. After receipt of HO_Req, the target service network generates response message to serving BS. On receipt of response message, serving BS sends MN_BSHO-RSP to the MN and an acknowledgment message to all other neighbouring BSs to complete the handoff preparation. The purpose of the MN_BSHO-RSP is to inform the MN on BSs it may connect itself during the actual handoff. Finally, handover indication message is sent by the MN to the serving BS before executing actual handoff. Now, MN disconnects old connections and ranges itself with the target BS [8] 
Further, a data path registration processes are executed between the target service access network and the anchor service network for bearer plane procedures. When the data path registration process is done, a HO_Complete message is sent from the target service network to the serving network to inform about completion of the handoff .

\subsection{Simulation Scenario}

Simulation scenario includes four MNs, out of which two are $\mathrm{BSs}$, one is a MN under one WiMAX network and another is a Correspondent Node $(\mathrm{CN})$ which is connected to both the BSs and named as CNO. Here the BS1 and BS2 are two stations of WiMAX network. No nodes are considered under BS2 for simplicity. Here the data transfer takes place between MN1 and the CN0 using CBR traffic system [9]. MN1 moves between the two networks.

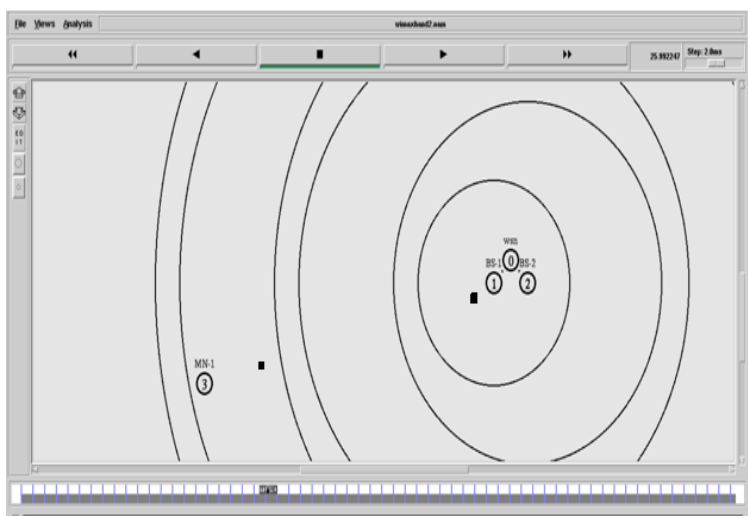

Fig 1: Simulation Scenario in ns2

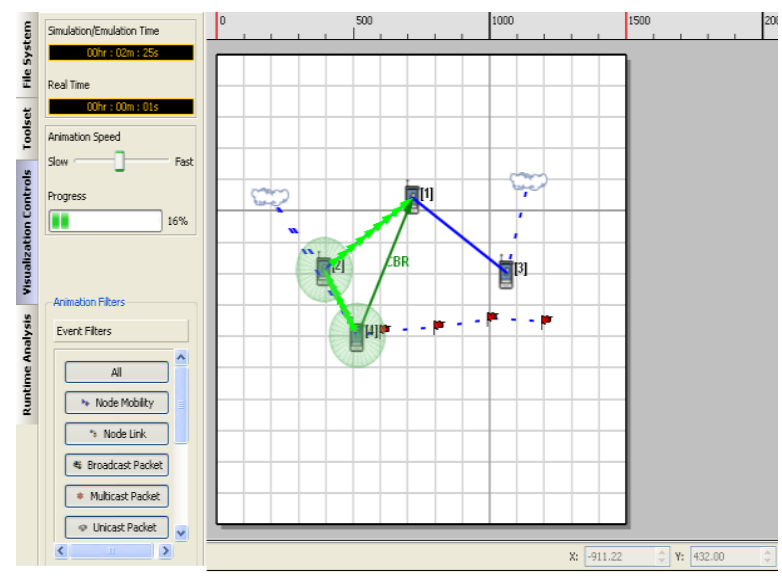

Fig 2: Simulation Scenario in QualNet

Simulation scenario of ns2, QualNet and simulation parameters are shown in Fig. 1, Fig. 2 and Table 1 respectively.
Table 1. Simulation Parameters

\begin{tabular}{|l|l|}
\hline Parameters & IEEE 802.16 \\
\hline Modulation & QPSK \\
\hline Area (in mtrs) & $1500 \times 1500$ \\
\hline Network & 2 Wireless Subnets \\
\hline Nodes & $4(\mathrm{BS}-2, \mathrm{MT}-1, \mathrm{CN}-1)$ \\
\hline Node Speed & $1 \mathrm{~m} / \mathrm{s}, 2 \mathrm{~m} / \mathrm{s}, 5 \mathrm{~m} / \mathrm{s}$ \\
\hline Simulation Time & $850 \mathrm{~s}, 430 \mathrm{~s}, 170 \mathrm{~s}$ \\
\hline Traffic Type & $\mathrm{CBR}$ \\
\hline Packets to Send & 500 \\
\hline Bit Rate & $64 \mathrm{kbps}, 256 \mathrm{kbps}, 512 \mathrm{kbps}$ \\
\hline
\end{tabular}

In order to ascertain the performance of Constant Bit Rate (CBR) streaming, impact on Packet Loss, End-to-End Delay and Throughput with different speeds and different data rates are evaluated. The CBR packet size transmitted between the MN and the network is 500 byte and CBR streaming is considered with three different data rates; $64 \mathrm{~Kb} / \mathrm{s}, 256 \mathrm{~Kb} / \mathrm{s}$ and $512 \mathrm{Mb} / \mathrm{s}$ $[10,11]$.

\subsection{Performance Analysis}

When the transfer is started initially the packets will be transferred from $\mathrm{MN}$ to $\mathrm{CN}$ via $\mathrm{BS} 1$. As the simulation time increases, MN starts moving away from BS1 and at a point moves out of coverage area of BS1. Further it senses the presence of better coverage by BS2 so handoff takes place and now the packets are sent from $\mathrm{MN}$ to $\mathrm{CN}$ via BS2. During this transfer $\mathrm{CN}$ is steady. As given in the Table 1, the data rates and speed of the MN is varied and graphs for packet loss, throughout and end-to-end delay are plotted using ns 2 and QualNet.

\subsubsection{Packet Loss}

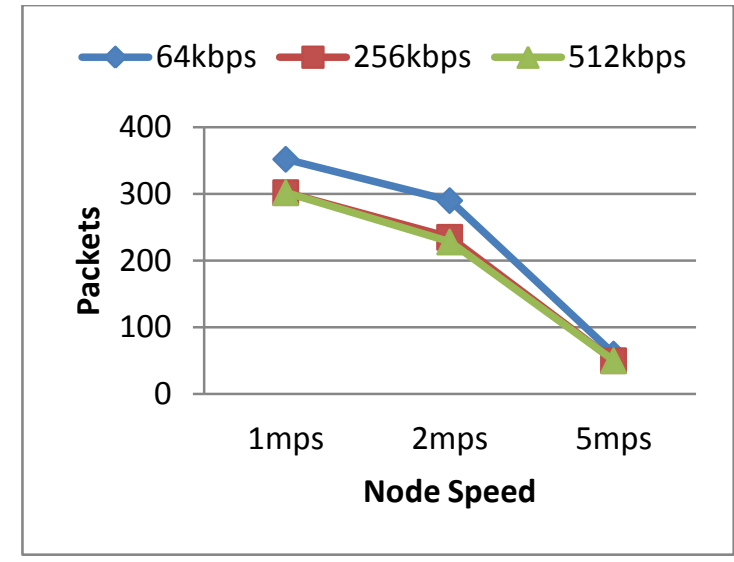

Fig 3: Packets Received by CN in NS2

For WiMAX to WiMAX handover the number of packets received at the $\mathrm{CN}$ for NS2 and QualNet are plotted in the graphs shown in Fig. 3 and Fig. 4 respectively. 


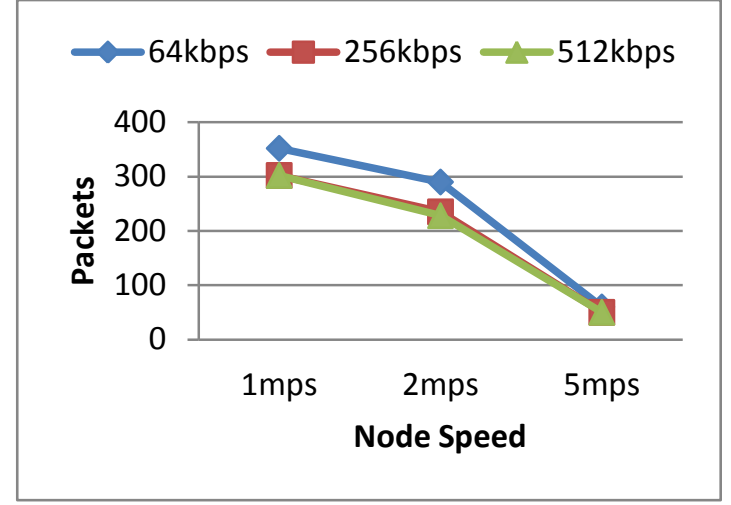

Fig 4: Packets Received by $\mathrm{CN}$ in QualNet

Graph shows that the number of packets received with the data rate of $64 \mathrm{kbps}$ is more than $256 \mathrm{kbps}$ and $512 \mathrm{kbps}$. For $256 \mathrm{kbps}$ and $512 \mathrm{kbps}$ the packet loss is same. Hence packet loss increases with increase in speed of the MN. It has also been observed that the packet loss in ns 2 is slightly more than that of the QualNet.

\subsubsection{Throughput}

Fig. 5 and Fig. 6 shows the graphs of throughput for WiMAX to WiMAX handoff in NS2 and QualNet respectively.

It is seen that the throughput remains constant when speed of $\mathrm{MN}$ changes from $1 \mathrm{mps}$ to $2 \mathrm{mps}$ but it decreases as the speed increases from $2 \mathrm{mps}$ to $5 \mathrm{mps}$. Throughput increases as the data rate increases. The throughput for $256 \mathrm{kbps}$ and $512 \mathrm{kbps}$ is nearly same in ns2 and is exactly same in QualNet.



Fig 5: Throughput (Bits/s) in NS2

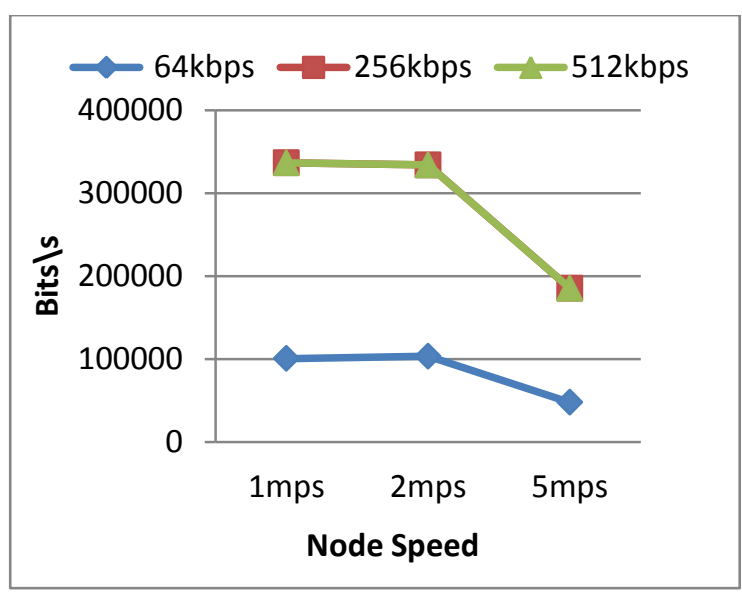

Fig 6: Throughput (Bits/s) in QualNet

\subsubsection{End-to-End Delay}

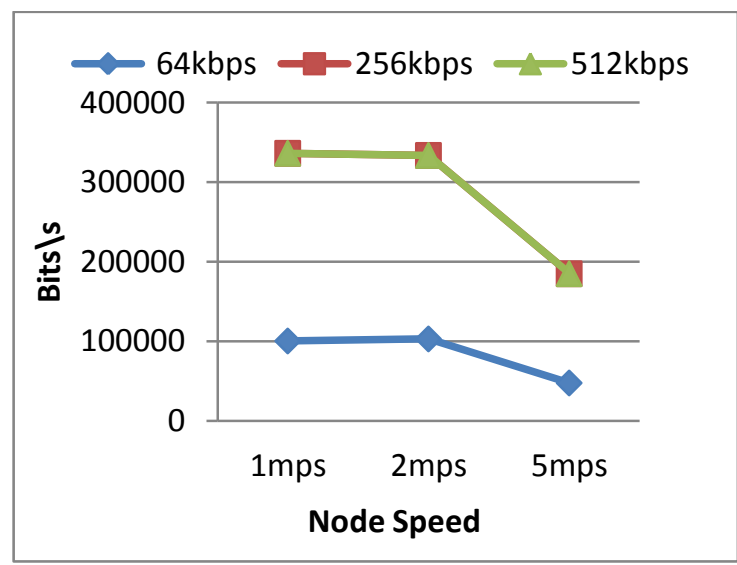

Fig 7: End-to-End Delay(s) in NS2

The time required for the packet delivery from source to destination during the handoff from WiMAX to WiMAX is plotted using ns2 and QualNet as shown in Fig. 7 and Fig. 8 respectively. End-to-End delay increases as the data rate increases. However, the change in the speed of the MN does not affect the End-to-End Delay remarkably. It is also observed that the delay in ns2 is less than QualNet.

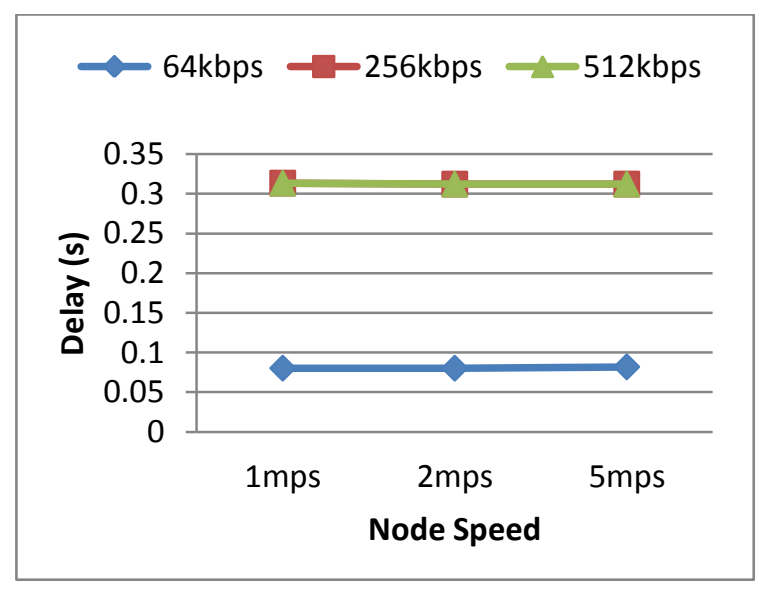

Fig 8: End-to-End Delay(s) in QualNet

\section{CONCLUSION}

Performance evaluation of QoS metrics during WiMAX to WiMAX HHO is carried out in ns2 and QualNet. These simulators have been designed to simulate large networks in order to analyze the mobility of a node during HHOs. It has been observed that both the simulators give nearly same results. Ns2 and QualNet proves to be a valuable and reliable tool to simulate such type of environments. Obtained results are an acceptable approximation to what could be expected in real case scenarios.

It is seen that when a mobile node moves away from coverage area of one network and finds better coverage in another network it undergoes handoff management procedures and starts communicating via newly detected PoA. Handoff process affects different QoS parameters such as Packet Loss, Throughput and End-to-End Delay. It is observed that the ratio of packet loss increases with the increase in the speed of $\mathrm{MN}$ and so is the throughput. This type of experimentation and analysis is extremely helpful to the students to understand the concept of handoffs through simulations. 
In future, the simulation scenario consisting of larger number of nodes can be generated and analyzed for heterogeneous access networks such as WiMAX, WiFi, UMTS and Bluetooth.

\section{REFERENCES}

[1] Mariem Zekri, Badii Jouaber, and Djamal Zeghlache. (2012) 'A review on mobility management and vertical handover solutions over heterogeneous wireless networks', Computer Communications, Vol. 35, pp. 2022-2068.

[2] Johann Marquez-Barja, Carlos T. Calafate, Juan-Carlos Cano, Pietro Manzoni.: An overview of vertical handover techniques: Algorithms, protocols and tools. In: Computer Communication, Vol. 34, pp. 985-997 (2011).

[3] Pranjali Bhandari, Poonam Jaswani, Shivani Singh, Sahana Bhosale, R.D. Daruwala, "Graphical Analysis of Parameters Related to Vertical Handover Optimization between Cellular Networks and WLANs," International Journal of Research in Wireless Sensor Networks IJRWSN Vol 01, Issue 01, April 2012.

[4] "The network simulator tool," ns-2, http://nsnam.isi.edu/nsnam/.

[5] "QualNet 5.0.2User's Guide", Scalable Network Technologies, Inc., March 2010.

[6] Kantubukta Vasu, Sumit Maheshwari, Sudipta Mahapatra, Cheruvu Siva Kumar, "QoS-aware fuzzy rule-based vertical handoff decision algorithm incorporating anew evaluation model for wireless heterogeneous networks," EURASIP Journal on Wireless Communications and Networking, doi:10.1186/1687-1499-2012-322, Oct 2012.

[7] BS Ghahfarokhi, N Movahhedinia. (2011) 'A contextaware handover decision based on user perceived quality of service trigger', Wirel. Commun. Mob. Comput. 11(6), 723-741.

[8] IEEE 802.16 WG, IEEE Standard for Local and Metropolitan Area Networks. Part 16: Air Interface for Fixed Broadband Wireless Access Systems, IEEE Std. 802.16-2004, October 2004.

[9] Hugo Marques, Jose Ribeiro, Paulo Marques, Jonathan Rodriguez, "Simulation of 802.21 Handovers Using ns-2", Journal of Computer Systems, Networks, and Communications", Vol. 2010, Article ID 794749.

[10] Sahana Bhosale and Dr. R.D.Daruwala, "Simulation of Vertical Handover between WiFi and WiMax and its Performance Analysis - An Installation Perspective”, IEEE INDICON Engineering Sustainable Solutions, Hyderabad, India, Dec 2011.

[11] Sahana Bhosale and Dr. R.D.Daruwala, "Experimental Analysis of Horizontal and Vertical Handovers in Wireless Access Networks using NS2", World Congress on Information and Communication Technologies (WICT), Mumbai, India, Dec 2011. 